Ehrhart, P., Haubold, H.-G. \& Schilling, W. (1974). Advanc. Solid State Phys. 14, 87-110.

EISENRIEGLER, E. (1971). Cryst. Lattice Defects, 2, 181-195.

HaUbOLD, H.-G. (1974). Rep. Kernforschungsanlage Jülich, JÜL-1090-FF.

Haubold, H.-G. \& Schilling, W. (1975). To be published.

Levelut, A. M. \& Guinier, A. (1967). In Small-Angle $X$-ray Scattering, edited by H. BRUMBERGER, pp. 351-371. New York: Gordon \& Breach.
Maier-Leibnitz, H. \& Springer, T. (1966). Ann. Rev. Nucl. Sci. 16, 207-262.

Rosenbaum, G., Holmes, K. C. \& Witz, J. (1971). Nature, Lond. 230, 434-437.

Schmatz, W. (1973). In Treatise on Materials Science and Technology, Vol. 2, pp. 105-229. New York: Academic press.

Trinkaus, H. (1972). Phys. Stat. Sol. (b) 51, 307-319.

Wombacher, P. (1972). J. Phys. E, 5, 243-245.

J. Appl. Cryst. (1975). 8, 183

\title{
A Lattice-Statics Approach to the Calculation of Diffuse X-ray Scattering by Interstitials in Metals*
}

\author{
By Roy BENEDEK $\dagger$ \\ Department of Materials Science and Engineering, Cornell University, Ithaca, New York 14850, U.S.A.
}

(Received 29 April 1974)

\begin{abstract}
The calculation of the intensity of diffuse X-ray scattering by interstitials involves the evaluation of a crystal structure factor which accounts for both the configuration of the interstitial atom and the distortion of the surrounding lattice. The lattice-distortion field surrounding an interstitial may be calculated using a Green's function lattice-statics approach described in earlier work [Benedek, R. \& Ho, P. S. (1973). J. Phys. F: Metal Phys. 3, 1285-1295]. In this approach, several atoms in the vicinity of the interstitial (the interstitial 'core') are treated formally on the same footing as the interstitial itself. Once the Green's function calculation of the lattice distortion field is performed the structure factor can be evaluated as follows. The contribution from the interstitial core is calculated explicitly using the coreatom displacements. The contribution from the remainder of the lattice is expressed in terms of ' $\mathrm{Kan}$ zaki forces' and certain 'virtual' displacements of core atoms, hoth of which are by-products of the Green's function calculation. Using this approach numerical calculations of diffuse scattering by selfinterstitial atoms in tungsten were performed. Based on the results, typical features of the Huang scattering, small-angle scattering, and scattering far from reciprocal-lattice points are discussed. The sensitivity of the calculations to the interatomic potential are also discussed.
\end{abstract}

* This work was supported by the U.S. Atomic Energy

Commission.

$\uparrow$ Present address: Argonne National Laboratory, 9700

South Cass Avenue, Illinois 60439.

J. Appl. Cryst. (1975). 8, 183

\section{Investigation of the Strain Field of Bismuth in Lead by Diffuse Elastic Neutron Scattering}

By E. Seitz, W. Schmatz and G. Bauer

Institut für Festkörperforschung der Kernforschungsanlage Jülich, Postfach 365, 517 Jülich, Germany (BRD)

AND W. JUST

Institut Laue-Langevin, Grenoble, France

(Received 29 April 1974)

The diffuse elastic neutron scattering due to distortion of the $\mathrm{Pb}$ host lattice around $\mathrm{Bi}$ substitutional atoms has been measured at the HFR in Grenoble. Two single crystals, with 2 and 4 at. \% Bi and with 100 and 110 orientations respectively, were used. The diffuse elastic scattering intensity is equivalent to the scattering of 0.05 and 0.1 at. \% of vacancies respectively. Using a wavelength of $4.3 \AA$ and a timeof-flight technique, the measuring range in reciprocal space was extended to $\kappa=2 \cdot 8 \AA^{-1}$ compared with $\kappa=1.8 \AA^{-1}$ of earlier measurements [Schumacher, H., Schmatz, W. \& Seitz, E. (1973). Phys. Stat. Sol. $(a), 20,109]$. The scattering patterns agree within the common $\kappa$ range. The improved resolution, however, shows that the 110 peak at $\kappa=1.8 \AA^{-1}$ observed earlier is more intense and has a smaller width than that described by the model calculations of Schumacher et al. These calculations assumed a distortion field around the $\mathrm{Bi}$ atoms caused by anisotropic forces acting on nearest neighbour $\mathrm{Pb}$ atoms. The new results require a more refined model. 